\title{
Renal myxoma: An unforeseen diagnosis
}

\author{
Rui Miguel Bernardino ${ }^{1}$, Luis Severo ${ }^{1}$, Luis Mascarenhas Lemos ${ }^{2}$, Luis Campos Pinheiro ${ }^{1}$ \\ ${ }^{1}$ Urology Department, Central Lisbon Hospital Center, Lisbon, Portugal; \\ ${ }^{2}$ Pathology Department, Central Lisbon Hospital Center, Lisbon Portugal.
}

\begin{abstract}
Summary Myxomas are rare tumours that can be found in many anatomical locations.
\end{abstract}

There are only 17 cases of renal involvement documented. Our case is an 85 year-old man followed in our consultation with recurrent hematuria after a transurethral resection of a bladder tumour. Evaluation with CT showed a solid lesion with $23 \times 18 \mathrm{~mm}$ partially obliterating the left inferior calyx. The patient underwent a left nephroureterectomy. Microscopic examination showed a mass within renal parenchyma adjacent to the renal pelvis composed of plump mildly atypical spindle cells distributed in a copious myxoid matrix.

Immunohistochemical staining for Vimentine, Pankeratin (AE1/AE3-), CD34, CD31 and smooth muscle actin were negative. With these histopathological and immunohistochemical findings, the case was diagnosed as renal myxoma.

KEY WORDS: Kidney; Cancer; Myxoma.

Submitted 2 March 2020; Accepted 15 March 2020

\section{INTRODUCTION}

Myxomas are rare benign tumours that can be found in many anatomical locations, such as skin, bones and heart. There are only 17 cases of renal involvement documented. We report a case of a renal myxoma in an old man with history of urothelial tumour, presenting the preoperative imaging evaluation as well as the pathological findings. The present article reports the second case of renal myxoma published in English Literature, that was associated with extensive haemorrhage and the first case of a renal myxoma in a patient with past history of urothelial tumour, which made the differential diagnosis even more difficult.

\section{Case presentation}

We present an 85-year-old man, followed in our Institution since January 2017 for bladder tumour.

He underwent transurethral resection of a left lateral wall bladder polyp whose anatomy revealed low grade noninvasive papillary urothelial (or transitional cell) carcinoma(pTa). After surgery the patient maintained haematuria. Follow-up cystoscopy (3 months after the surgery) did not show lesions suggestive of relapse. The patient had an emergency in June 2017 due to massive haematuria with clots and with repercussion in the hemogram, hemoglobin of $6.5 \mathrm{~g} / \mathrm{dL}$, receiving two units of erythrocyte concentrate. Evaluation with Computed Tomography (CT) showed a solid lesion with $23 \times 18 \mathrm{~mm}$ partially obliterating the left inferior calyx (Figures 1, 2). At this time, surgery was recommended for this patient not only due to malignant fea- tures on CT but also for his past history of urothelial tumour. He underwent a left laparoscopic nephroureterectomy. After one year of follow-up, the patient was well and completely symptom-free and the annually routine CT didn't show relapse. He died one year and a half after the surgery from myocardial infraction.

\section{Imaging findings}

Ultrasound: Renal ultrasound revealed a solid nodular lesion in the middle third of the left kidney with about 23 x 18 mm. Right Kidney had no alterations.

CT: CT scan confirmed a solid lesion partially obliterating the lower left calyx, suspected of urothelial neoplasia (Figures 1, 2). MRI was not done in our case, as the patient had a past history of urothelial tumour, and CT was high suspicious for urothelial neoplasia.

Renogram: He performed renogram showing normal renal function (Left Kidney - 57\% and Right Kidney 43\%).

\section{Pathological findings}

Lesion within renal parenchyma adjacent to the renal pelvis, circumscribed, somewhat lobulated, composed of plump mildly atypical spindle cells distributed in a copious myxoid matrix (Figure 3). A subset of these cells had irregular atypical nuclei, although the atypia was more prominent adjacent to the ulcerated surface of the lesion and may therefore be reactive in nature. There is no significant pleomorphism, mitotic activity or necrosis (Figure 4). The lesion cells were positive for vimentin, negative for pankeratin, multifocally positive for smooth muscle actin, while were negative for S-100 protein, CD34, CD31 and pancytokeratin. With the above findings, the lesion was labelled as an atypical myxoid spindle cell neoplasm, consistent with renal myxoma. The lesion has expansive growth conditioning extension and erosion of the adjacent pyelocalyceal structures and no extracapsular extension was documented.

\section{Discussion}

There is no reports of specific clinical presentation for renal myxoma and, due to its rarity, renal myxoma is often mislead with other malignant lesions (1). When there were clinical manifestations, flank pain was the most common presenting symptom reported in the 17 cases (2). It is known so far that there is no invasion, metastasis or tumour recurrence (3). In our renal myxoma case, the patient had concomitant past history of

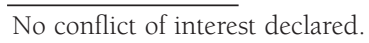




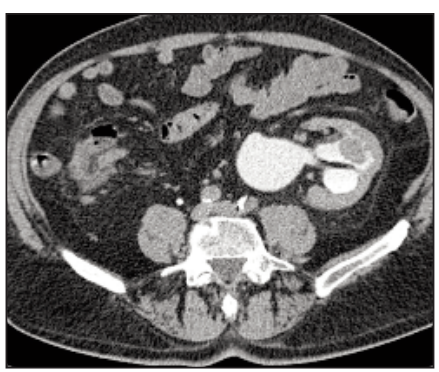

Figure 1.

CT scan (excretory phase) - confirmed a solid lesion partially obliterating the lower left calyx of about $23 \times 18 \mathrm{~mm}$, suspected of urothelial neoplasia.

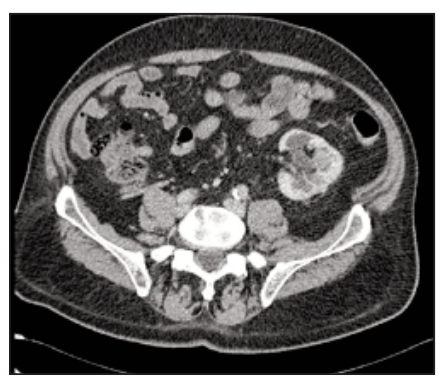

Figure 2.

Same lesion as shown in figure 1 but in nephrographic phase.

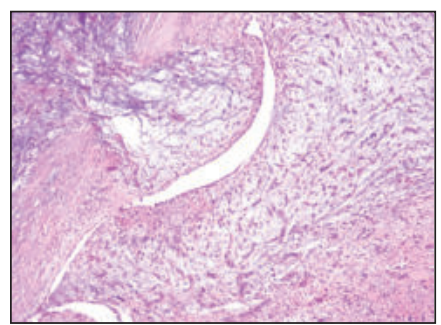

Figure 3.

(100x) - H\&E stain

Benign mesenchymal lesion, intraparenchymatous, composed of spindle cells, with a slightly eosinophilic cytoplasm and light and focal cytological atypia, arranged around vessels or scattered on a

background of myxoid and haemorrhagic stroma.

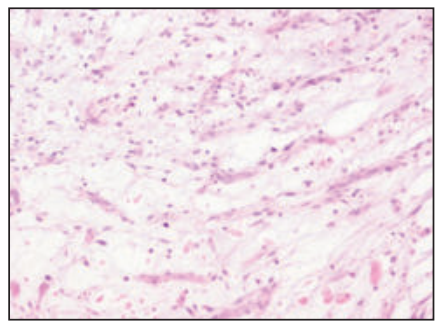

Figure 4.

(400x) - H\&E stain No mitosis or necrosis is documented.

urothelial bladder tumour, which made the differential diagnosis with a urothelial neoplasia even more difficult. The origin of renal myxoma from the renal capsule is less common than parenchymal renal myxoma (1).

Fibroblast is the originary cell of myxoma.

Macroscopically, this tumour shows a gelatinous gross appearance due to production of glycosaminoglycan and it is well-defined (3). Microscopically, the spindle-shaped cell can be seen in the myxoid stroma, and no mitotic activity is seen. In all reported cases, the tumour cells stained positive for vimentin and in most cases negative for S-100 protein, pancytokeratin and CD34.

Renal myxoma is a large heterogeneous mass in imaging exams, predominantly hyperechoic in ultrasonography (US) and hypodense in CT. Myxomas characteristics in imaging is a relatively regular, multilobulated and welldefined mass, only displacing the adjacent structures without invading them. Therefore, pre-operative imaging cannot confidently distinguish myxoma from other renal masses and therefore histopathological confirmation is the only reliable way of doing so. In this context, what is the role of percutaneous biopsy? In general, a biopsy should be performed only to avoid unnecessary or incorrect treatment. Appropriate laboratory and diagnostic imaging tests should be exhausted first. Only when these steps prove to be inconclusive should a patient be subjected to the risks, discomfort, and expense of a biopsy (4). In our case, as the patient had a history of urothelial bladder tumour, and the CT was high suspicious for urothelial neoplasia, a percutaneous biopsy was not indicated.

As the radiological characteristics are very close to those of malignancy, they must be treated as malignant tumors, with the patient being surgically treated. For most cases reported, nephrectomy was the treatment of choice due to suspicion to malignancy. Although imaging is required to differential diagnosis, pathological evaluation is needed to differentiate a myxoma from malignancy. Advanced imaging models such as CT guided biopsy can help us to get closer to the diagnosis, but for definitive diagnosis, we need immunohistochemistry evaluation (5). Tumour enucleation of the myxoma would be enough not only for diagnosis but also for treatment, and overall prognosis of this disease is good (1).

\section{Conclusions}

- Renal myxomas are extremely rare mesenchymal tumours of the kidney.

- The radiological characteristics are very close to thoseof malignancy, therefore they must be treated as malignant tumors.

- Pathological evaluation is needed to differentiate a myxoma from malignancy.

- If the diagnosis of the lesion before surgery is renal myxoma for sure, the best treatment option would be, tumour enucleation.

\section{REFERENCES}

1. Shah A, Sun W, Cao D. Myxoma of the kidney associated with hemorrhage. Indian J Surg. 2013:75(Suppl. 1):S480-3.

2. Suthar KS, Vanikar AV, Patel RD, et al. Renal myxoma-A rare variety of benign genitourinary tumour. J Clin Diagn Res. 2015; 9:ED11.

3. Hakverdi S, Görür S, Yaldiz M, et al. Renal myxoma: case report and review of the literature. Turk J Urol. 2010; 36:318-21.

4. Young M, Leslie SW. Renal Biopsy. (Updated 2019 Jun 17). In: StatPearls (Internet). Treasure Island (FL): StatPearls Publishing; 2019 Jan-.Available from: https://www.ncbi.nlm.nih.gov/books/ NBK470275/

5. Salehipour M, Geramizadeh B, Dastgheib N, Makarem A, Asadollah Poor A, Taheri N. Renal myxoma, a case report and review of the literature. Urol Case Rep. 2018; 23:21-22.

\section{Correspondence}

Rui Miguel Bernardino, MD (Corresponding Author) ruimmbernardino@gmail.com

Luis Severo, MD - luis.severo@sapo.pt

Luis Mascarenhas Lemos, MD - luisalbuquerquermascarenhas@gmail.com Luis Campos Pinheiro, MD - luiscampospinheiro@gmail.com

Centro Hospital de Lisboa Central, EPE - Serviço de Urologia, Alameda de Santo António dos Capuchos, 1169-050 Lisboa (Portugal) 